\title{
Entrelacs
}

Cinéma et audiovisuel

Hors-série $n^{\circ} 4$ | 2016

Paysages en séries

\section{Paysage Apocalyptique en série, ou comment les zombies reconfigurent l'espace télévisuel}

Paysage Apocalyptique en série

\section{Olivier Ammour-Mayeur}

\section{(2) OpenEdition}

\section{Journals}

Édition électronique

URL : http://journals.openedition.org/entrelacs/2158

DOI : $10.4000 /$ entrelacs. 2158

ISBN : $2261-5482$

ISSN : 2261-5482

Éditeur

Éditions Téraèdre

Édition imprimée

Date de publication : 1 novembre 2016

ISSN : 1266-7188

\section{Référence électronique}

Olivier Ammour-Mayeur, « Paysage Apocalyptique en série, ou comment les zombies reconfigurent l'espace télévisuel », Entrelacs [En ligne], Hors-série n 4| 2016, mis en ligne le 06 février 2018, consulté le 04 mai 2019. URL : http://journals.openedition.org/entrelacs/2158 ; DOI : 10.4000/entrelacs.2158

Ce document a été généré automatiquement le 4 mai 2019.

Tous droits réservés 


\title{
Paysage Apocalyptique en série, ou comment les zombies reconfigurent l'espace télévisuel
}

\author{
Paysage Apocalyptique en série
}

\author{
Olivier Ammour-Mayeur
}

1 Les paysages des villes et des campagnes proposées par les séries consacrées aux Zombies n'offrent pas seulement le reflet d'une apocalypse liée à une pandémie qui annihile l'espèce humaine. Ils proposent en fait une tentative de représentation de ce que le capitalisme, poussé à outrance, réserve à l'espèce humaine dans un avenir plus ou moins proche.

2 Bien qu'il ne soit pas possible en un seul article de faire le tour de la question sur le rapport entre l'idée de paysage et l'espace humain mis en scène dans les séries portant sur la question zombie, les prouesses techniques visuelles mises en œuvre afin de rendre l'atmosphère de ces villes désertées et de ces campagnes désolées méritent toute l'attention. Ce sera donc à travers quelques paysages exemplaires des trois séries Zombies qui dominent actuellement l'espace télévisuel du genre - The Walking Dead, Fear The Walking Dead et $Z$ Nation - que j'aimerais proposer une analyse de ce que l'apocalypse révèle de nos sociétés hypermodernes ${ }^{1}$.

3 Il faut d'abord noter que, quelle que soit la série, certains paysages s'y trouvent exploités de façon récurrente. Ceux-ci se déclinent selon trois modalités: l'opposition entre dedans/dehors - opposition qui, on le verra, ne tient plus en réalité -, l'inversion des polarités entre sécurité et danger du point de vue de la vie quotidienne et, enfin, une dislocation du rapport privé/public lié à la maison - au sens anglais du home, «lieu » dans lequel l'humain est censé retrouver ses marques après avoir évolué dans «l'espace indéfini » du monde extérieur².

4 Les séries américaines zombies construisent ainsi un monde qui semble s'inscrire en exact antagonisme avec les séries familiales qui les précèdent et dont Jean Mottet analyse les positions en ces termes : 
La télévision s'occupe d'abord de dresser la topographie du territoire proche, qu'on s'approprie par d'infimes cheminements, à la portée d'un enfant. Car cet enclos rassurant est le neighborhood de l'enfant, personnage clef du genre, qui ne fait encore qu'entrevoir à travers fentes et interstices, la sauvagerie du dehors. (моттет, p. 47)

6 La série Zombie, prenant le contrepied exact de ce dispositif familial "sécurisant", envoie ses « enfants » - ici entendu au sens large de tous ceux qui se retrouvent face à «la sauvagerie du dehors" - sur les routes, afin que ceux-ci tentent de "dresser la topographie du territoire» le plus lointain. En d'autres termes, les enjeux se trouvent bouleversés au plus haut degré. Retournés parfois même, comme on peut le dire d'un gant dont le dedans deviendrait le dehors et inversement.

7 Afin d'analyser ces transformations, je propose d'examiner de façon transversale trois "espaces" qui reviennent régulièrement à l'écran dans les trois séries zombies dominantes : la route, la maison - ainsi que sa version religieuse, l'église - et enfin le seuil ; ce dernier devenant un espace fondamental de ces séries, dans lesquelles il indique de façon encore plus marquée l'effondrement des valeurs dialectiques qui fondent le rapport humain à son environnement.

\section{La route}

Disons-le d'emblée, la série Zombie - à l'instar des productions cinématographiques du même genre - offre un exemple type de ce que Freud appelle Unheimlich. Le psychanalyste écrit sur le sujet que :

9 Le mot allemand unheimlich est manifestement l'antonyme de heimlich, heimisch (du pays), vertraut (familier), et l'on est tenté d'en conclure qu'une chose est effrayante justement pour la raison qu'elle n'est pas connue ni familière. Mais, il est évident que n'est pas effrayant tout ce qui est nouveau et non familier; la relation n'est pas réversible. On peut seulement dire que ce qui a un caractère de nouveauté peut facilement devenir effrayant et étrangement inquiétant ; parmi les choses revêtant un caractère de nouveauté, quelques-unes sont effrayantes, mais certainement pas toutes. Au nouveau, au non-familier doit d'abord s'ajouter quelque chose, pour qu'il devienne étrangement inquiétant. (FREUD, pp. 215-216, italiques dans le texte)

Ce concept de Unheimlich induit deux postures possibles en ce qui nous concerne: d'une part, la situation des personnages confrontés à une apocalypse Zombie sort bien des normes du «familier » et, d'autre part, Unheimlich se rapportant à ce qui appartient à la "maison", les séries Zombies prennent l'exact contre-pied de cette notion. Le lieu, opposé ici à l'espace - selon la distinction opérée par Roger Silverstone (1999), et discutée par Jean Mottet -, se trouve littéralement déterritorialisé. Plus de « chez soi », plus de lieu Heimlich qui puisse abriter et protéger de l'espace du dehors.

11 Ce sentiment se trouve renforcé si l'on reprend les propos de E. Jentsch cités par le psychanalyste sur Unheimlich et selon qui :

12 L'un des stratagèmes les plus sûrs pour provoquer aisément par des récits des effets d'inquiétante étrangeté [...] consiste donc à laisser le lecteur dans le doute quant à savoir s'il a affaire, à propos d'un personnage déterminé, à une personne ou par exemple à un automate, et ce de telle sorte que cette incertitude ne s'inscrive pas directement au foyer de son attention, afin qu'il ne soit pas amené à examiner et à tirer la chose aussitôt au clair, vu que, comme nous l'avons déjà dit, cela peut aisément compromettre l'effet affectif spécifique. (Ibid., p. 224) 
(1)

l'axiologique de la route offre un vecteur particulièrement efficace. En effet, les personnages de ces séries se trouvent littéralement jetés sur les routes, sans plus aucun point d'ancrage familier subsistant. Dans les trois séries la route devient, à la fois et tour à tour, espace d'apprentissage - comme on le disait du Bildungsroman du XIX ${ }^{\mathrm{e}}$ siècle -, espace de survivance - il semble plus aisé d'échapper aux attaques zombies en restant en plein air que confiné dans un lieu fermé et, enfin, espace de réappropriation de l'espacemême. Les personnages de ces séries, privés de technologie avancée permettant de voyager en avion ou sur de longues distances, doivent redécouvrir l'Amérique et ses « dangers " à l'instar de leurs ancêtres cow-boys des Westerns américains. Comme leurs ancêtres découvraient le Far-West et les pièges de la «Wild Life », les personnages des séries Zombies sont appelés à essayer de ré-apprivoiser l'espace envahi par les mortsvivants ${ }^{4}$.

15 L'analogie entre survivants de l'apocalypse Zombie et cow-boys est explicitement mise en scène dans la première saison de The Walking Dead où Rick Grimes, ancien " deputy sheriff " d'une petite ville proche d'Atlanta, se voit contraint de partir à la (re)conquête de « l'Ouest » à cheval, à travers un paysage (re)devenu hostile à l'homme. Par suite, Rick est présenté comme une figure perdue dans l'immensité qu'il est amené à traverser. Il doit alors tenter de se (re)familiariser avec l'Unheimlich du monde qui l'entoure après son coma de plusieurs semaines. Ainsi le spectateur, accompagnant Rick dans sa quête de survivants et dans l'apprentissage de sa (sur)vie, devine à l'instar du personnage ce qu'ont été les lieux traversés et reconnaît certaines coordonnées géographiques et cartographiques des espaces représentés à l'écran. Pourtant rien n'y est plus ce qu'il était : la civilisation humaine n'est que ruines et le territoire doit être réévalué à l'aune de la nouvelle donne épidémiologique et des dangers qu'elle implique.

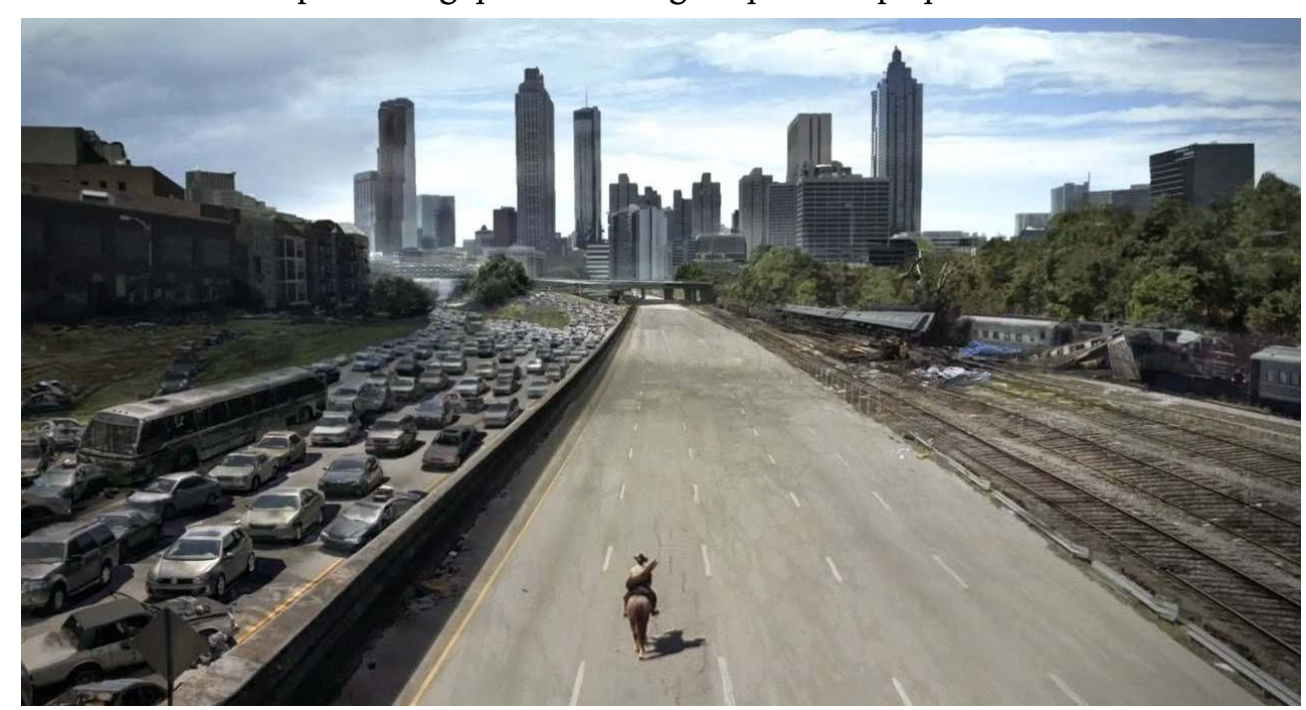

Fig 1 : The Walking Dead (saison 1)

16 On peut citer André Bazin qui, analysant la notion de Western, emploie tout un lexique cinématographique qui s'adapte parfaitement aux séries Zombies : 
17 Ainsi trouve-t-on à la source du western une éthique de l'épopée et même de la tragédie. Épique, le western l'est, croit-on généralement, par l'échelle surhumaine de ses héros, l'ampleur légendaire de leurs prouesses. Billy le Kid est invulnérable comme Achille, et son revolver, infaillible. Le cow-boy est un chevalier. Au caractère du héros correspond un style de mise en scène ou la transposition épique apparaît dès la composition de l'image, sa prédilection pour les vastes horizons, les grands plans d'ensemble rappelant toujours la confrontation de l'Homme et de la Nature. Le western ignore pratiquement le gros plan, presque le plan américain, il affectionne en revanche le travelling et le panoramique qui nient le cadre de l'écran et restituent la plénitude de l'espace. (BAzIN, p. 225)

18 Évidemment la question de la "plénitude de l'espace " américain en territoire Zombie tend à ne plus avoir la même valeur, d'expansion et de conquête par l'Homme, que dans le western. Cependant, la métaphore, déplacée sur le Zombie conquérant l'espace, reste valide, de même que la quête des survivants pour se réapproprier le territoire national. Par ailleurs, le rapport au Western n'est pas non plus sans ambiguïté puisque la distinction entre les "good guys» et les "bad guys» n'est plus aussi tranchée. Les protagonistes principaux sont souvent amenés à commettre des actes qui ébranlent la notion éthique de " héros ». Comme le répète régulièrement Rick - et que ne démentirait aucun personnage des trois séries ici étudiées - «It's them, or it's us! ». Là où le positionnement éthique engagé devient problématique, c'est que ce syntagme - déployé en de multiples variantes au fil des saisons - ne concerne pas seulement les Zombies mais bien tout autre groupe croisé par celui de Rick, que ces nouveaux survivants se présentent comme d'agressifs antagonistes ou non.

19 Pour preuve de l'ambivalence axiologique des espaces traversés, lorsque Rick part à la recherche de ses proches à Atlanta - censée être un havre protecteur contre l'épidémie fulgurante - il découvre, et le spectateur avec lui, que sa population a été rayée de la carte. Ainsi, les villes, toujours considérées comme surpeuplées et peu sûres, tombent les premières sous l'invasion épidémiologique puisqu'aucune barrière ne permet d'y enrayer le processus de contagion. Cette représentation de la ville en tant que lieu pathogène est aussi employée dans Fear The Walking Dead, où la première saison montre comment l'absence de clôtures concrètes, liée au mouvement de panique général, accélère la progression de la pandémie et ce d'autant plus que les gens ignorent littéralement ce qu'il se passe ${ }^{5}$.

20 Là où l'épopée Zombie s'autonomise quant à la trajectoire suivie par ses héros c'est lorsque, contrairement à la poursuite de « lignées arborescentes » (comme les appellent Deleuze et Guattari) que l'on peut déceler dans les récits épiques classiques, ces nouveaux cow-boys du XXI siècle doivent composer avec des déplacements rhizomatiques et non plus topographiques :

21 Le rhizome procède par variation, expansion, conquête, capture, piqûre. À l'opposé du graphisme, $d u$ dessin ou de la photo, à l'opposé des calques, le rhizome se rapporte à une carte qui doit être produite, construite, toujours démontable, connectable, renversable, modifiable, à entrées et sorties multiples, avec ses lignes de fuite. (DELEUZE \& GUATTARI, p. 32)

En outre, cette notion de rhizome met en relief le parallèle existant entre l'avancée Zombie et celle des survivants : dans un cas comme dans l'autre l'avancée " procède par variation, expansion, conquête, capture, piqûre». Les Zombies n'ont que faire des frontières et des "chemins" tracés par la technologie humaine qui les précède. Ils avancent comme l'épidémie qu'ils incarnent et s'infiltrent dans toutes les strates de 
l'espace offert à leurs mouvements. De leur côté, les survivants doivent progresser de façon à éviter non seulement les hordes de zombies mais aussi les autres groupes de survivants, souvent hostiles. Autrement dit, les chemins tout tracés n'offrent plus la sureté nécessaire aux déplacements prévus. Les rencontres impromptues imposent que la carte suivie reste toujours "démontable, connectable, renversable, modifiable » et surtout «à entrées et sorties multiples » en cas de repli nécessaire.

En particulier, les Highways ne connectent plus un point $\mathrm{A}$ à un point $\mathrm{B}$, car elles ont été les premières prises d'assaut au moment de l'éclatement de la pandémie. Elles se retrouvent dès lors jonchées de voitures abandonnées, qui empêchent tout déplacement rapide. Leur utilité première s'en trouve ainsi bannie, et leur usage perdu. Elles deviennent même le lieu par excellence du danger suprême : leurs axes de rapidité étant coupés, elles deviennent le lieu des mauvaises rencontres, ce que mettent en scène les trois séries, chacune à sa façon.

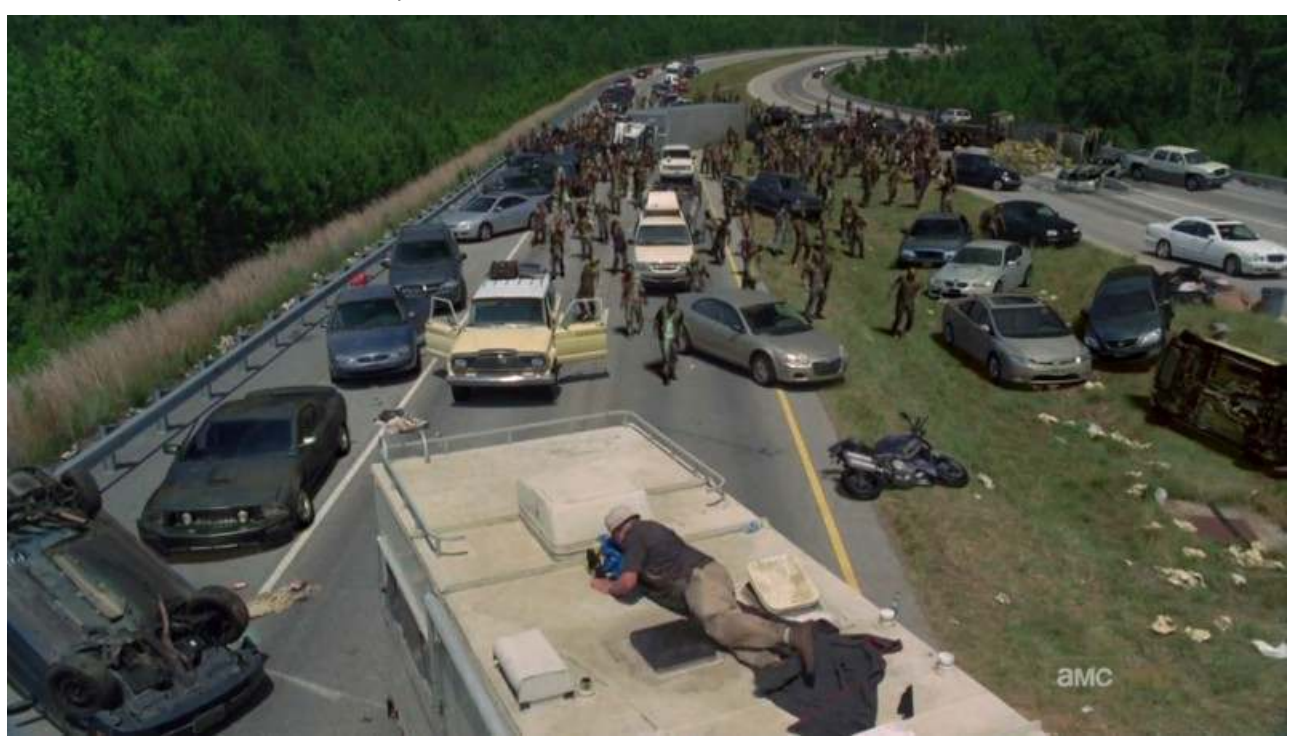

Fig 2 : The Walking Dead (saison 1)

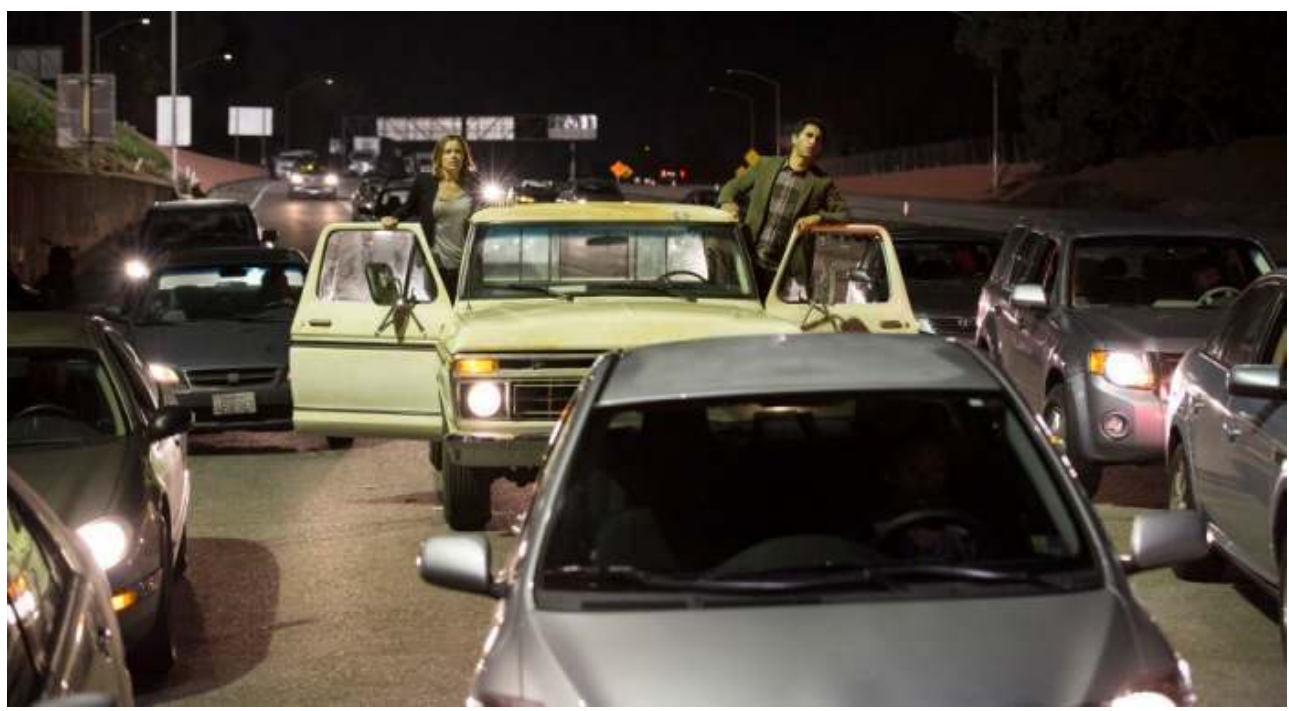

Fig 3 : Fear The Walking Dead (saison 1) 


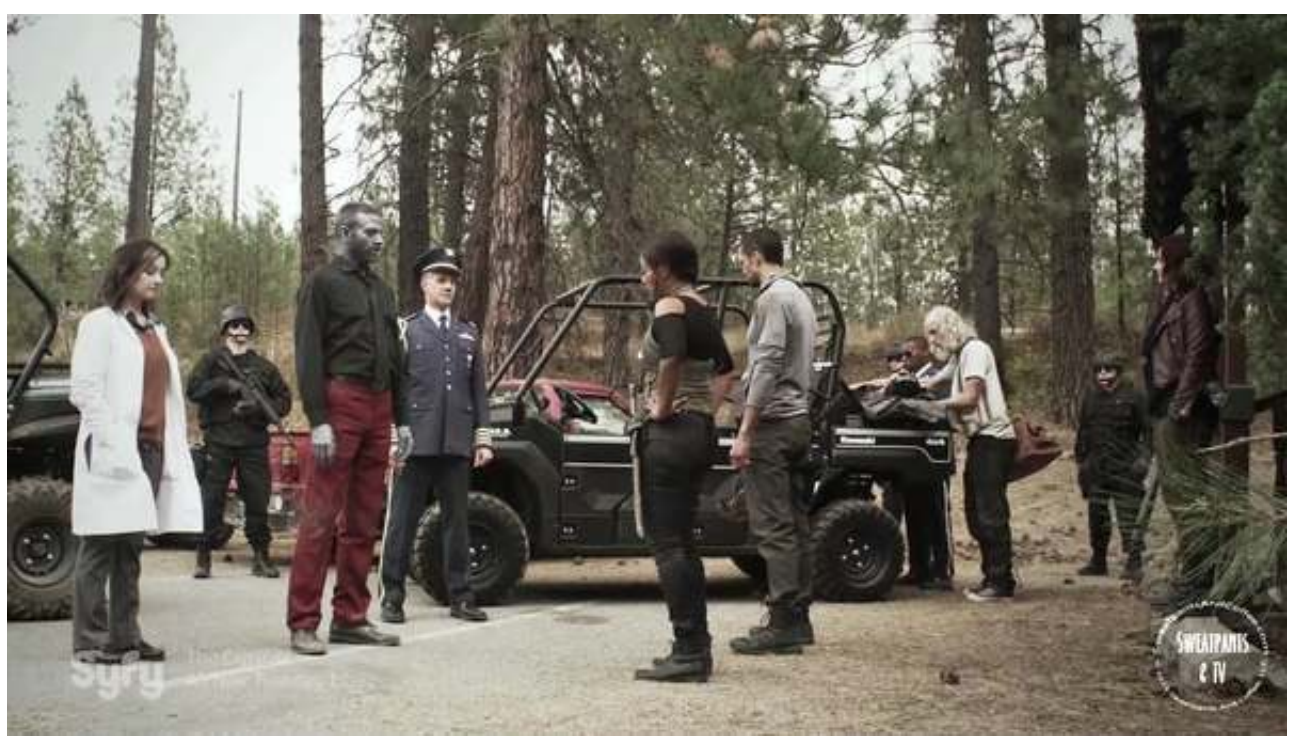

Fig 4 : Z Nation (saison 2)

Ce n'est donc pas pour rien si, là encore dans les trois cas qui nous occupent, l'image de la route est couplée à celle de la forêt. $\mathrm{Si}$, dans le contexte qui est le nôtre la route - emblème de civilisation - semble plutôt s'opposer à la sauvagerie des bois, en territoire zombie les pôles s'inversent ou, pour dire mieux, se mêlent en une forme hybride. D'une part, la route représente l'espace permettant d'avancer et de garder l'œil ouvert sur les possibles mauvaises rencontres malgré les lenteurs qu'elle impose aux personnages mais, d'autre part, celle-ci, trop souvent encombrées et offrant peu - pas - d'endroit où s'abriter en cas de besoin, ne permet pas - plus - de se déplacer de façon fluide et sécurisée.

De même, la forêt présente une double figure de Janus. D'une part, lieu de chasse ou de repos pour l'être humain dit "civilisé », elle devient lieu du chassé, les Zombies et les survivants antagonistes pouvant y surgir à chaque instant. D'autre part, la forêt, bien qu' $a$ priori hostile, permet d'offrir un lieu de repli immédiat, ainsi qu'un espace de survie possible, mais non permanent, en cas d'urgence.

\section{La maison, l'église}

Dans ce paysage post-apocalyptique, que devient le lieu de l'intime par excellence - la maison? Par "maison" ici nous entendons le "home», selon le terme anglais, par opposition à « house », qui reste un lieu extérieur à soi. En l'espèce, la dialectique dedansdehors semble se trouver renversée, voire même pulvérisée. En effet, si dans les séries familiales la maison représente le lieu qui importe, celui où la famille se retrouve et travaille ensemble à résoudre les conflits et trouver des solutions, dans les séries zombies ce lieu de rassemblement n'est plus. En tout cas il n'apparaît plus selon les mêmes stratégies narratives : la maison n'est plus le lieu que l'on rejoint pour « se retrouver », ou trouver le réconfort nécessaire face à l'apocalyptique épidémie mais, bien au contraire, le lieu qu'il faut fuir et laisser derrière soi si l'on ne veut pas être contaminé par les membres de sa famille déjà touchés ou même être pris au piège par une horde de passage.

En d'autres termes, la maison post-apocalyptique reflète celle que nous voyons se développer de plus en plus ces dernières années. Lieu de rassemblement d'individualités, 
l'espace familial n'est plus le cocon rassurant qu'on a bien voulu nous faire croire qu'il était. Peuplée de femmes battues (comme Carol de The Walking Dead), d'enfants maltraités (Daryl de The Walking Dead) et de couples divorcés ou en instance de divorce (Fear The Walking Dead, The Walking Dead), la maison n'est plus le havre de paix que les séries familiales se sont complues à nous représenter. Par suite, cet espace, initialement entendu comme celui du rassemblement familial comme amical, est devenu le lieu de tous les dangers. Ceci se révèle d'autant plus marquant dans Fear The Walking Dead et The Walking Dead, du fait qu'il est établi dans la série mère et sa prequel que tout le monde est porteur du virus et que la morsure Zombie ne change rien, en réalité, à la situation sanitaire : tous les morts sont appelés à se relever. Ainsi le virus Zombie métaphorise tout un ensemble de dangers potentiels et implique - parce que l'apocalypse qu'il engendre concerne absolument tout le monde - toutes les classes sociales comme toutes les configurations familiales. À sa manière, l'apocalypse Zombie propose donc une déconstruction de la notion de maison/home, entendue en son sens familial, ainsi qu'une mise en relief de l'importance des recompositions familiales. En effet, aucun groupe de survivants ne parvient à subsister sans en passer par la paradoxale reconstruction d'un foyer sans demeure, famille en restructuration permanente et sans domicile fixe. De fait le nomadisme est redevenu dans ces séries le mode de vie de l'homo sapiens sapiens.

The Walking Dead pousse d'ailleurs l'ironie en mettant en scène le caractère parfaitement dystopique des quelques lieux où semble se réélaborer un semblant de "vivre ensemble »: Woodbury (saison 3) est en réalité une sorte de dictature qui ne dit pas son nom; le "Gouverneur " se révèle être, au fil des épisodes, un pervers manipulateur psychopathe et sadique; et enfin Alexandria s'avère fort mal préparée à défendre son territoire dès qu'il s'agit de passer aux choses sérieuses. Les premières rencontres avec l'extérieur s'achèvent systématiquement dans un bain de sang des survivants. De même, alors qu'il semblerait, par contraste, que la vie en prison que choisissent les survivants du groupe de Rick Grimes puisse tenir ses promesses de jardin de Candide (Voltaire), le conflit avec le « Gouverneur » montre très rapidement les limites de ce projet. Plus aucun endroit ne tient lieu d'espace privé - même en terme d'espace communautaire - et le concept-même de " propriété privée » n'a plus de sens. Ainsi, une prison nettoyée de ses morts-vivants devient, contre toute attente (selon nos « règles » de propriétés actuelles), un espace focalisant toutes les convoitises.

Si j'ai pris le parti d'aborder dans un même ensemble la maison et l'église, c'est que la «maison de Dieu » occupe une place centrale dans chacune des séries abordées ici. Pour résumer quelque peu la teneur de leur message, on pourrait dire que la «maison de Dieu ", au même titre que la maison individuelle, n'occupe plus la place sanctifiée qu'elle pouvait avoir dans les séries familiales plus conventionnelles ${ }^{6}$. Dans Fear The Walking Dead, c'est à l'intérieur d'une église abandonnée que l'épidémie débute et c'est depuis cet endroit qu'elle se propage. Devenue un abri pour junkies qui squattent ses murs pour se shooter, l'église est ainsi présentée d'emblée comme abandonnée non seulement des humains, mais aussi de sa figure tutélaire divine qui ne semble plus se préoccuper de ce qui se passe sur le sol normalement sacré de ses lieux de culte. 


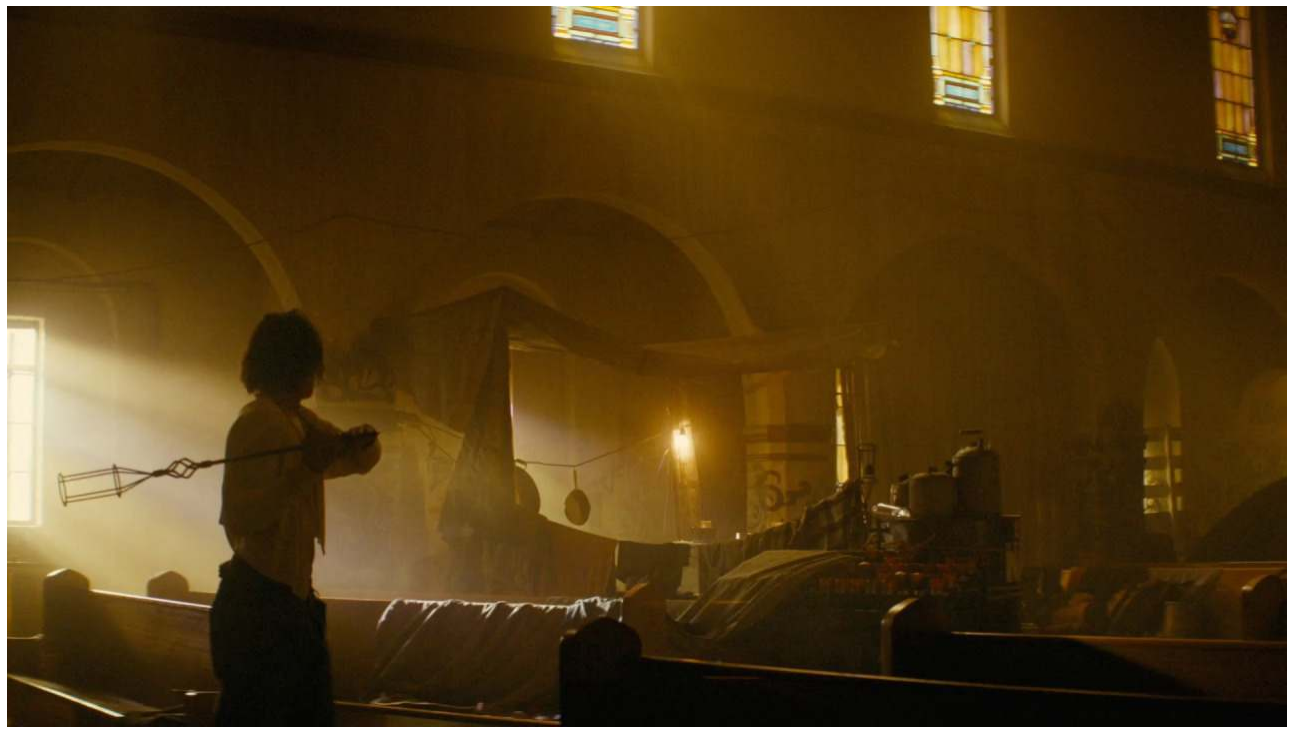

Fig 5 : Fear The Walking Dead (saison 1, épisode 1)

30 Dans The Walking Dead, plusieurs églises se présentent sur le trajet aléatoire du groupe de survivants. Dans chaque cas, elle s'est transformée en lieu d'agonie, soit que les personnes de la communauté qui s'y sont réfugiées ont fini par s'entre-dévorer, soit que le prêtre en charge du culte leur ait barré la porte, faisant voler en éclat l'un des principes de son sacerdoce : le devoir de protection vis-à-vis de ses ouailles. Dans un cas comme dans l'autre, l'église a perdu son statut sacré et d'asile: les prières n'ont visiblement pas été entendues. Il en va de même pour la maison familiale qui, perdant son statut de havre et de protection, pousse les êtres sur les routes, dans un périple nomade aux pièges multiples.

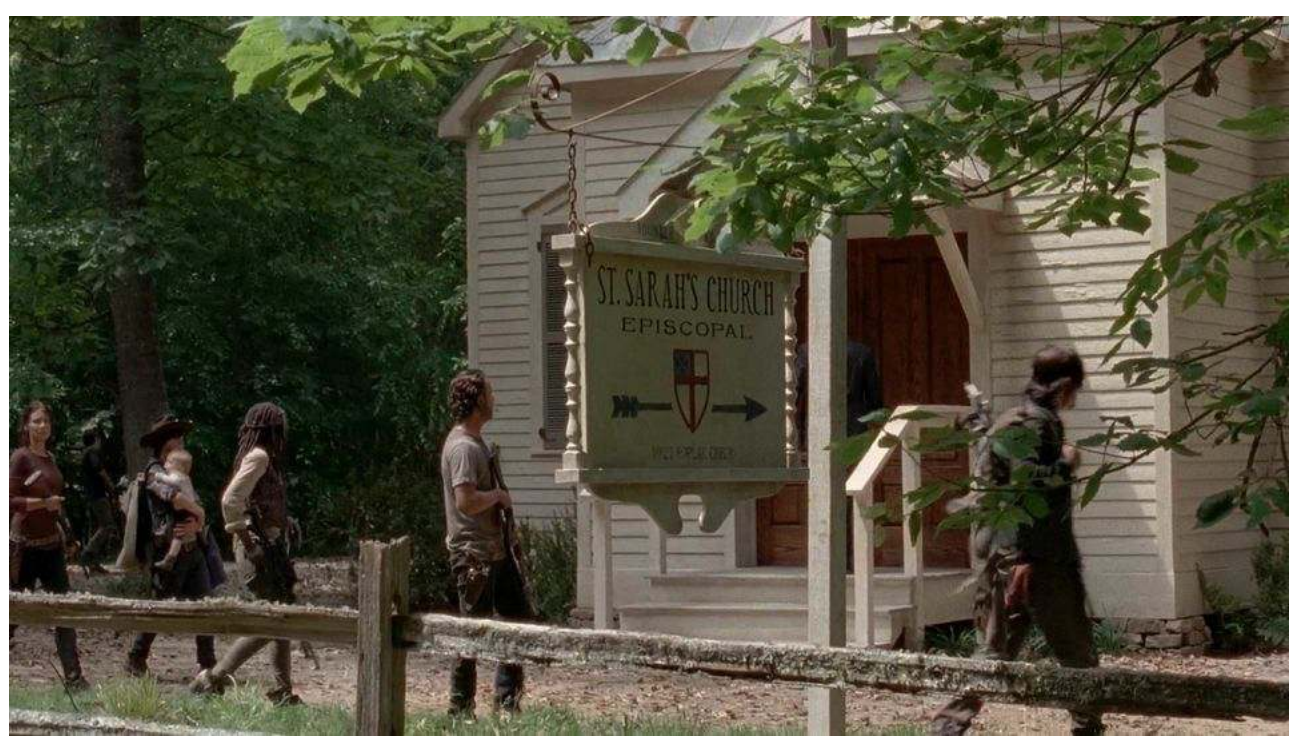

Fig 6 : The Walking Dead (saison 4)

31 Qu'il s'agisse du « picket fence » des quartiers WASP, analysé par Jean Mottet (op. cit.), ou de l'enceinte sacrée de l'église communautaire, plus aucun des lieux symboles de sécurité et de ressourcement n'a de sens en territoire zombie. 


\section{Le seuil}

En ce qui concerne le seuil, lieu neutre par excellence, autrement dit ne-uter ${ }^{7}$ à la fois dedans et dehors, autrement dit ni dedans ni dehors - c'est à travers un texte de Bachelard qu'il semble opportun d'en introduire la signification. Le philosophe écrit ainsi au sujet de la grotte que :

Dès le seuil on peut sentir une synthèse d'effroi et d'émerveillement, un désir d'entrer et une peur d'entrer. C'est ici que le seuil prend ses valeurs de décision grave. (BACHELARD, p. 200)

L'épidémie Zombie ayant littéralement fait exploser les notions d'intime et d'extime, tout seuil, même autre que celui des grottes, instille ce double sentiment antagoniste. Qu'y a-til derrière cette porte? Dans ce tunnel que les personnages doivent emprunter? De l'autre côté de la barricade qu'il faut parvenir à dépasser? Étant donné que les Zombies ne sont pas les seuls prédateurs que les survivants doivent affronter, chaque épisode des séries Zombies apporte son lot de franchissement de seuils divers - dans un sens comme dans l'autre - afin d'entretenir le sentiment Unheimlich chez le spectateur. Les lieux familiers ne semblent plus que l'ombre d'eux-mêmes. En d'autres termes, tout geste de dépassement, de franchissement de seuil, implique chez le personnage - et le spectateur qui suit ses mouvements - l'effroi préventif de ce qu'il risque de trouver de l'autre côté et en même temps l'émerveillement anticipateur d'avoir trouvé, peut-être, un lieu plus propice au repos. Qui plus est, à l'instar on l'a vu de la route et de la maison, ces seuils ne se présentent plus comme des lieux forcément salvateurs contre la pandémie Zombie, ni même contre les attaques d'autres groupes humains.

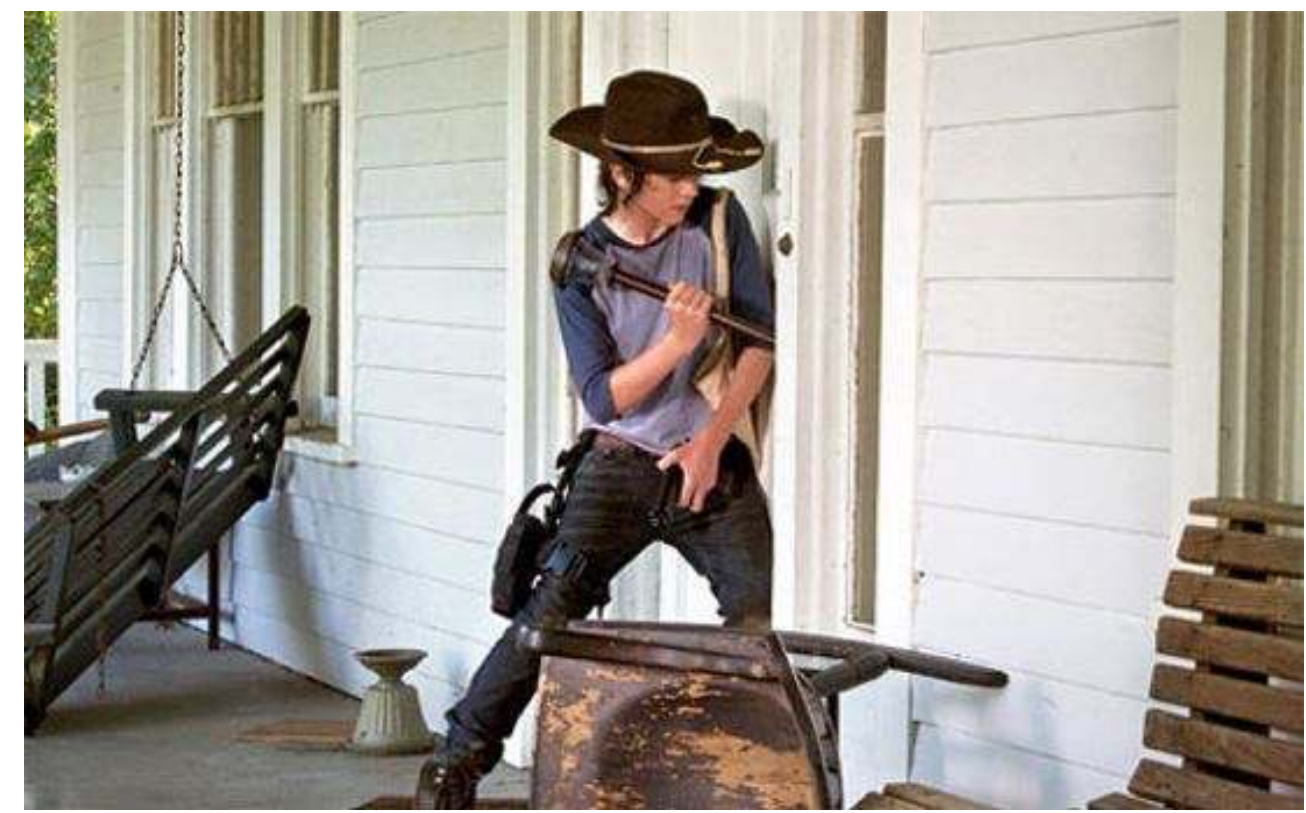

Fig 7 : The Walking Dead (Saison 3) 


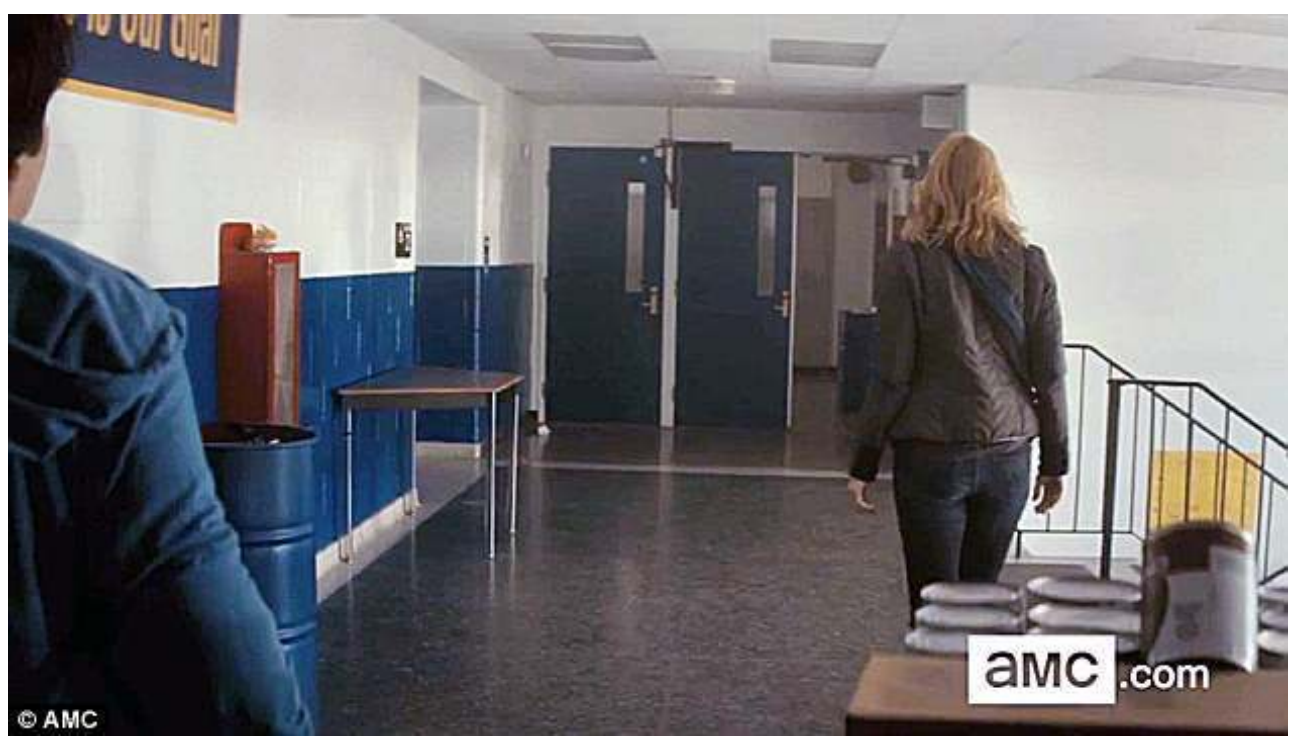

Fig 8 : Fear The Walking Dead (Saison 1, épisode 1)

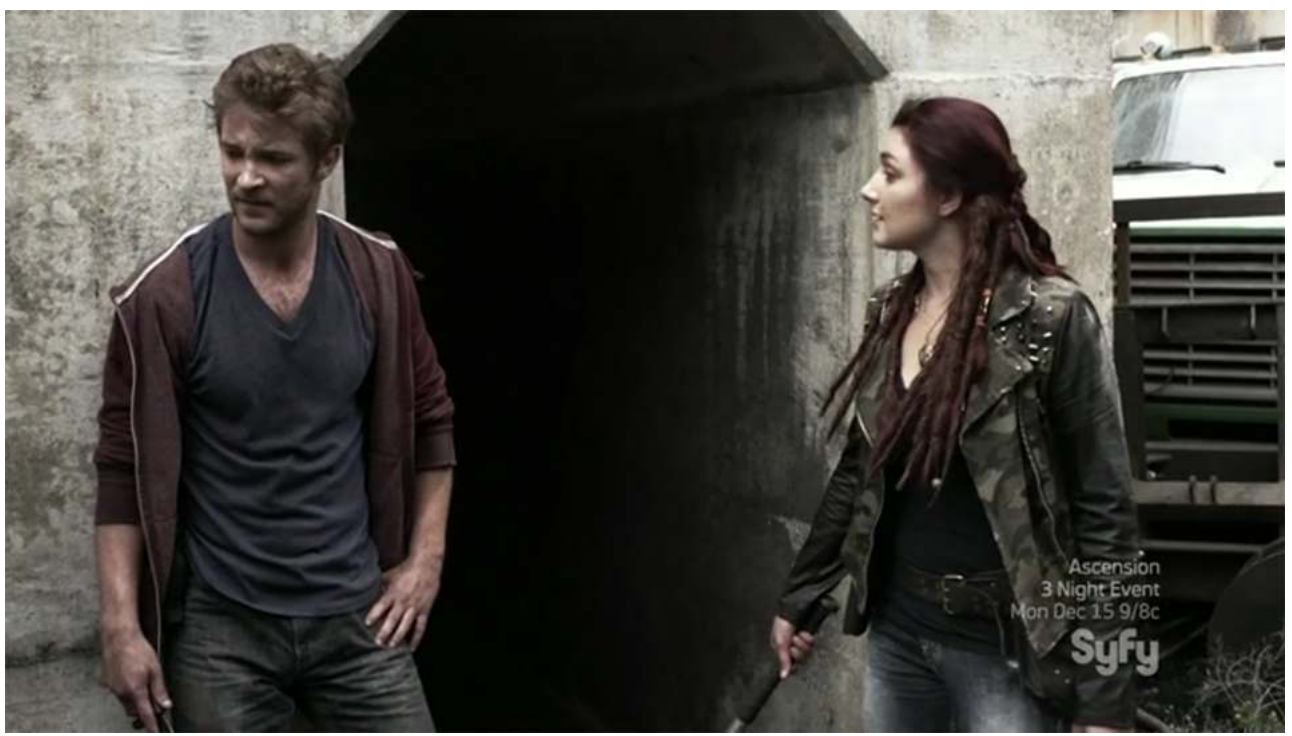

Fig 9 : Z Nation (Saison 1)

Si l'on reprend l'exemple de la Prison des saisons 3 et 4 de The Walking Dead, il apparait de façon assez explicite que la situation des personnages métaphorise littéralement la problématique de la contagion virologique et/ou bactérienne. Entourée de deux grillages considérés comme de haute sécurité, la prison peut faire penser à une cellule biologique, les grillages représentant alors la membrane protectrice de son noyau - ici le bâtiment carcéral. Or, à l'instar de ces cellules qui nous constituent, ces clôtures apparaissent finalement bien plus poreuses qu'elles n'en avaient l'air au départ. Dans The Walking Dead, après les attaques sanglantes du gouverneur de Woodbury, le groupe de Rick est forcé de quitter la prison dont les protections ont finalement lâché et qui est submergée par les Zombies. 


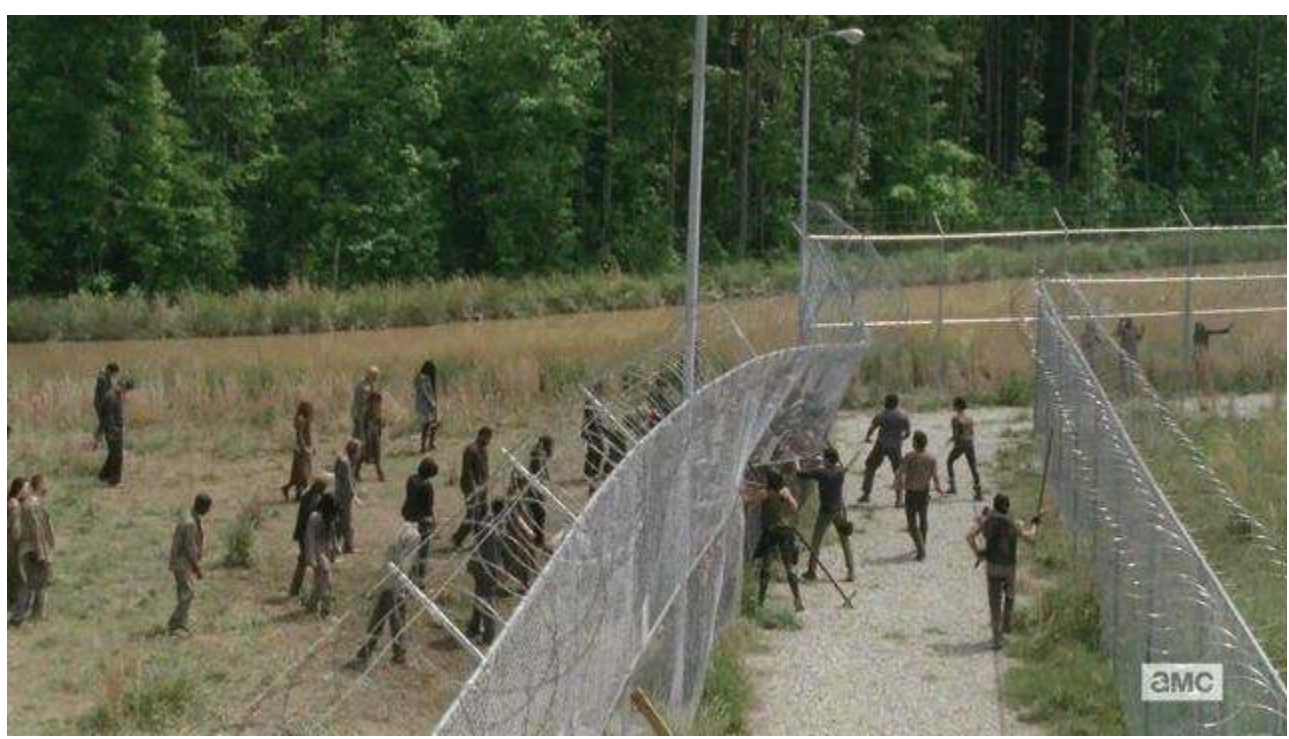

Fig 10 : The Walking Dead (saison 4)

Ce rapprochement entre prison et cellule organique est mis en scène de façon explicite à plusieurs reprises, notamment lorsque les protagonistes tentent de tuer les Zombies à travers les grilles parce qu'ils se font trop nombreux autour de l'enceinte protectrice. Les notions de «dedans » et « dehors » prennent ainsi une toute autre connotation : le virus Zombie colonise les corps de même que ces corps, ces Zombies, se répandent dans toutes les zones disponibles au niveau terrestre en parvenant même, par leur nombre, à franchir des seuils qui semblaient jusqu'alors hermétiques et sûrs.

Les franchissements de seuils ainsi redéfinis par la problématique Zombie mettent dès lors au jour l'aspect purement fantasmatique de ces notions de dedans/dehors ou d'intime/extime. En effet, dans le contexte de réalité sociologique qui est le nôtre, les séries Zombies ne font que souligner le fait que nos conditionnements mentaux, par rapport à ces couples dialectiques, tendent à abolir la porosité intrinsèque que ces termes recouvrent en réalité. Ainsi, ces grilles pénitentiaires rejouent, tout en la déplaçant quelque peu, la métaphore - encore une - du «picket fence» des séries télévisées familiales conventionnelles. En l'occurrence, ces grilles qui séparent le dedans et le dehors mettent en relief le fait qu'en une fraction de seconde le dedans protecteur peut se transformer en piège mortel: l'invasion Zombie impliquant, le seuil une fois franchi, métamorphose du lieu providentiel en fatale souricière.

Dès lors, si le seuil opère comme lieu intermédiaire entre l'espace extérieur et le lieu intérieur, il n'en demeure pas moins que ce couple terminologique se voit lui-même battu en brèche par ce que vivent les personnages à l'écran. On pourrait donc reformuler la relation entre les deux notions ainsi : en territoire Zombie, ce n'est plus l'intime qui chaque jour tente l'aventure vers l'espace extime, mais bien l'extime qui tente, par tous moyens, de coloniser le lieu de l'intime et de le muer en espace extime. Cette abolition des frontières signe la fin non seulement de l'intime mais surtout de la propriété : de soi, de son environnement personnel, de son point d'ancrage référentiel. C'est bien évidemment ce qui peut arriver de pire à l'homme blanc, censé représenter le nec plus ultra de la colonisation territoriale.

Dans Fear The Walking Dead, les «picket fences » n'existent plus sur le devant des maisons, dans le quartier de " white collars » où habitent les Clark-Manawa. Cependant, les jardins 
arrières sont délimités par des barrières en bois, dont certaines laissent même place à des espaces intermédiaires - simples espaces vides - à travers lesquels il est possible de se déplacer. Là encore, ces barrières mettent clairement en évidence les problèmes qui se posent en temps de contagion fulgurante. Ces étroites sentes, hors-lieux en quelque sorte puisque n'appartenant à personne, permettent à certains personnages de fuir en temps utile mais empêchent certains autres - moins bien informés - d'échapper à leurs prédateurs. Par suite, le seuil réel devenu sans objet, il devient, de façon de plus en plus marquée au fil des saisons des différentes séries, la ligne de démarcation - tout à fait imaginaire, et pourtant de plus en plus prégnante - entre le dedans que représente le groupe de survivants et son dehors : tout le reste.

\section{Conclusion}

Pour conclure, il semble important de souligner pourquoi ces séries - très américanocentrées, puisqu'elles jouent la carte de l'absence quasi-totale des moyens de communication avec l'extérieur - parviennent à rencontrer un tel succès international. Tout d'abord - et il s'agit là d'un banal constat - les séries américaines se diffusent massivement dans le monde depuis les années 60 , ce qui a permis à une grande partie du globe de se familiariser avec la géographie américaine, même lorsque celle-ci s'avérait de carton-pâte. Surtout, l'apocalypse Zombie ne hante pas uniquement l'imaginaire américain. Le réchauffement climatique, les crises épidémiques diverses et de plus en plus virulentes - sans oublier leur corollaire : la résistance de plus en plus massive des virus aux antibiotiques - ont touché tous les continents.

41 À n'en point douter, ces peurs sanitaires, couplées avec les nouvelles angoisses liées au terrorisme depuis le 11 septembre 2001, ainsi qu'aux dangers du nucléaire dont Tchernobyl et Fukushima représentent les derniers avatars en date, ont profondément marqué les esprits, quelle que soit leur culture princeps. En d'autres termes, bien qu'américaines sur nombre de plans, les séries Zombies ont un pouvoir cathartique déterritorialisé - à l'instar du virus qu'elles mettent en scène - puisque la route, la maison/l'église et le seuil sont des notions ancrées dans l'idée du paysage quotidien. Ces lieux, devenus non-lieux des territoires Zombies, entrent dès lors en écho immédiat avec le téléspectateur, lequel aimerait savoir comment il réagirait et organiserait son nouvel espace de vie s'il était, à la place des personnages, confronté à une épidémie réelle.

En réalité, l'épidémie Zombie existe déjà bel et bien puisque, poussé hors de ses conventionnelles habitudes, le spectateur projette dans son propre quotidien les différents périls traversés pour lui par les personnages des séries télévisées. À leur façon, ces séries ne placent pas le spectateur devant un monde fictif, elles proposent en fait un reflet de notre monde en son virtuel devenir.

\section{Références}

Bachelard, Gaston. La Terre et les rêveries du repos. Paris : José Corti, 1948.

Bazin, André. Qu'est-ce que le cinéma ? Paris : Éd. du Cerf, 1985, rééd. 2005.

Deleuze, Gilles et Guattari, Felix. Mille Plateaux. Capitalisme et schizophrénie 2. Paris : Éd. de Minuit, 1980. 
Dell'Agnese Elena. « Post-Apocalypse now : Landscape and environmental values in The Road and The Walking Dead », Geographica Polonica, Volume 87, Issue 3, 2014.

Freud, Sigmund. «L'inquiétante étrangeté » [1919] (trad. B. Féron), in L'Inquiétante étrangeté et autres essais, Paris : Gallimard, 1985, rééd. Folio « Essais », 1988.

Gruber, Eberhard. «Quant au neutre », in Calle-Gruber Mireille. Du féminin. Grenoble/Québec : PUG/Le Griffon d'Argile, 1992.

Kennedy, Tiànna, Kennedy, Christina et Kennedy, Mélisa. "Science Fiction/Fantasy Films, Fairy Tales and Control : Landscape Stereotypes on a Wilderness to Ultra-urban Cotinuum", in Harper Graeme \& Rayner Jonathan (Eds.). Cinema and Landscape. Bristol/Chicago : Intellect Books, 2010. pp. 283-296.

Mottet, Jean. Série télévisée et espace domestique. La Télévision, la maison, le monde. Paris :

L'Harmattan, 2004.

Overbey, Erin. “'The Walking Dead' returns”, The New Yorker, 12 October 2012. Article accessible en ligne : http://www.newyorker.com/books/double-take/the-walking-dead-returns.

Silverstone, Roger,.Television and Everyday Life. London/New York : Routledge, 1999.

\section{NOTES}

1. Les trois séries ont rencontré des records d'audience aux Etats-Unis : la première saison (2014) de Z Nation a terminé avec une moyenne de 1,42 millions d'auditeurs, tandis que la première saison (2015) de Fear The Walking Dead a connu une moyenne de 11,2 millions de spectateurs. Mais, c'est sans conteste The Walking Dead qui prend la première marche du podium, avec 17,3 millions de téléspectateurs lors de sa cinquième saison (2014-2015).

2. D'emblée il semble important de signaler l'article de Elena Dell'Agnese (2014). Mes analyses rejoignent certaines de ses propositions, cependant, mon étude portant sur un corpus quelque peu autre, ses conclusions diffèrent.

3. Bien entendu, la plupart des moyens de transport restent présents - voitures, camions, bus, bateaux, avions -, et les personnages s'en servent autant que possible. Cependant, le manque d'essence se fait de plus en plus sentir, et, dans la plupart des cas, les routes sont tellement encombrées de véhicules abandonnés que les déplacements ne se font plus ni en ligne droite, ni de façon continue. Les embuches sont nombreuses qui forcent les personnages à effectuer leurs déplacements par à-coups. Sans compter les mauvaises rencontres, qui s'accompagnent régulièrement de vol de véhicule, d'essence, etc. Les séries exploitent ainsi l'angoisse de l'auditeur-consommateur, en s'appuyant sur certaines projections statistiques qui prédisent l'épuisement des stocks de pétrole d'ici 30 ans.

4. Plusieurs auteurs traitant de The Walking Dead ont établi ce parallèle avec le Western. Pour plus de détails, on peut se référer à l'article en ligne de Erin Overbey, consulté le 10/03/2016 : http:// www.newyorker.com/books/double-take/the-walking-dead-returns.

5. La série Zombie reconduit, en parallèle avec le mythe du Far-West, la dichotomie établie aux États-Unis au XIX ${ }^{\mathrm{e}}$ siècle, entre ville et banlieue. Comme le souligne l'article de Christina, Tianna \& Melissa Kennedy : "The promotional image of the surbub initially was as 'a haven from the city's whirl'» p. 289. 
6. Parmi les séries familiales les plus connues, on retiendra: Little House on the Prairie (La Petite maison dans la prairie), NBC, 1974-1983 ; The Cosby Show, NBC, 1984-1992 ; Who's the Boss? (Madame est servie), ABC, 1984-1992 ; Full House (La Fête à la maison), ABC, 1987-1995, et son sequel Fuller House (La fête à la maison, 20 ans après), Netflix, 2016-présent ; My So Called Life (Angela, 15 ans), ABC, 1994-1995; Party of Five (La Vie à cinq), FOX, 1994-2000; Seventh Heaven (Sept à la maison), The WB, The CW, 1996-2007 ; Gilmore Girls, The WB, 2000-2007 ; The Fosters, ABC, 2013-présent.

7. Pour une analyse de cette notion, voir Eberhard Gruber. "Quant au neutre », », in Calle-Gruber Mireille. Du féminin. Grenoble/Québec : PUG/Le Griffon d'Argile, 1992.

\section{AUTEUR}

\section{OLIVIER AMMOUR-MAYEUR}

Olivier Ammour-Mayeur est actuellement maître de conférences en littérature à ICU (International Christian University) de Tokyo. Il obtient son doctorat en 2002 (Université Paris 8) puis son HDR en littérature comparée à l'Université Sorbonne Nouvelle - Paris 3 en 2014. L'inédit de son HDR porte sur les représentations littéraires et filmiques des bombardements de Hiroshima et Nagasaki. Il est également chercheur associé de l'équipe LLA-CRÉATIS (Université Toulouse 2). 